\title{
Heat shock protein 90 regulates neutrophil Mac-1 expression and extravascular recruitment in acute pancreatitis related with lung injury
}

\author{
Mohammed Yousif Merza ${ }^{1 *}$
}

\begin{abstract}
Background and objective: Severe acute pancreatitis (AP) is associated with infiltration of leukocytes and tissue necrosis but the cellular signaling mechanisms controlling organ damage in the pancreas remain elusive. Heat shock protein 90 (HSP 90) is a potent regulator of specific cellular processes effectuating pro-inflammatory activities. Herein, we examined the role of HSP90 signaling in acute pancreatitis.

Methods: Pancreatitis was provoked by infusion of taurocholate into the pancreatic duct in C57BL/6 mice. Animals were treated with radicicol $(60 \mathrm{mg} / \mathrm{kg})$ prior to induction of pancreatitis.
\end{abstract}

Results: Administration of radicicol significantly decreased the taurocholate-induced increase in serum amylase, pancreatic neutrophil infiltration, acinar cell necrosis and edema formation in the pancreas related with lung injury. In addition, inhibition of heat shock protein 90 reduced the MPO levels in the pancreas and lung in response to taurocholate challenge. However, treatment with radicicol had no effect on taurocholate-provoked formation of macrophage inflammatory protein-2 (MIP-2) in the pancreas. Interestingly, Heat shock protein 90 inhibition abolished neutrophil expression of Mac-1 in mice with pancreatitis. Moreover, radicicol markedly decreased MIP-2-induced Mac-1 upregulation in isolated neutrophils in vitro, suggesting a direct role of heat shock protein 90 in regulating Mac-1 expression in neutrophils. Finally, inhibition of heat shock protein had no direct effect on secretagogue-induced activation of trypsinogen in pancreatic acinar cells in vitro.

Conclusion: These results demonstrate that heat shock protein 90 signaling plays a significant role in acute pancreatitis by regulating neutrophil infiltration and tissue injury via expression of Mac-1 on neutrophils. Thus, our findings not only elucidate signaling mechanisms in pancreatitis or lung injury but also suggest that heat shock protein might constitute a novel target in the management of severe AP related with lung injury.

Keywords: Amylase, Chemokines, Inflammation, Leukocytes, and Pancreas.

\section{Introduction}

Acute pancreatitis (AP) presents with a wide range of disease severity ranging from simple and transient pain to local and systemic complications. ${ }^{1}$ Due to the limited understanding of the underlying pathophysiology, management of patients with severe AP poses a major challenge to clinicians, which is largely limited to supportive therapies. Today, there is no effective method to predict the severity and outcome of AP. The literature suggests that trypsinogen activation, inflammation and impaired microvascular perfusion are integrated components in the pathophysiology of pancreatitis. ${ }^{2}$ Knowing that activation of trypsinogen appears to be an early and transient process, inflammation in the pancreas persists longer and might be a more rational target for treatment. ${ }^{2}$ Leukocyte accumulation is a hallmark of inflammation and several reports have shown a key role of leukocytes in the development of AP. ${ }^{3}$

${ }^{1}$ Department of Clinical Analysis, College Pharmacy, Hawler Medical University, Erbil, Iraq.

* Correspondence: mohammed.merza@hmu.edu.krd 
The extravasation process of leukocytes comprises multiple sequential steps mediated by specific adhesion molecules, such as P-selectin, Mac-1 and LFA-1. ${ }^{4}$ Tissue navigation of leukocytes is orchestrated by secreted chemokines. ${ }^{5}$ CXC chemokines, such as macrophage inflammatory protein-2 (MIP-2), stimulate extravascular recruitment of neutrophils. CXCR2 is the high affinity receptor on murine neutrophils for MIP-2 and KC and it has been shown that CXCR2 is critical in supporting neutrophil infiltration in the pancreas. ${ }^{6}$ Although, the role of specific adhesion molecules and chemoattractants in leukocyte infiltration in the pancreas is relatively well known, the understanding of the signaling pathways coordinating pro-inflammatory actions in AP is limited. Trauma and infection trigger multiple signaling cascades that converge on specific transcription factors controlling gene expression of pro-inflammatory substances. This signal transmission is predominately regulated by intracellular kinases phosphorylating down-stream targets. ${ }^{2}$ Targeting Hsp90 protects against intestinal inflammation and leakage and might be a useful strategy to ameliorate intestinal failure in polymicrobial sepsis.inflammatio. ${ }^{7}$ In several disease models, including pancreatitis, heat shock proteins (HSPs) are chaperone proteins that protect living cells against injury-inducing stimuli. However, dysregulated expression of HSPs has been observed in various disease conditions including cancer. Inhibition of HSP especially HSP 70 expression in pancreatic cancer cells leads to caspase dependent apoptotic cell death and it has been shown as a novel therapeutic modality for pancreatic cancer. ${ }^{8}$ Based on these considerations, we hypothesized that heat shock protein signaling might play a role in severe AP. We used an experimental model of severe AP with lung injury in mice based on retrograde infusion of taurocholate in the pancreatic duct and interfered with HSP 90 activity by administration of radicicol.

\section{Methods}

Animals

All experiments were conducted using C57BL/6 male mice, weighting $20-25 \mathrm{~g} \mathrm{(6}$ to 8 weeks) purchased from Taconic. The animals were maintained under a $12 \mathrm{~h}$ light/ dark cycle in a climate at $22^{\circ} \mathrm{C}$ and fed water and standard chow ad libitum. Mice were anesthetized by intraperitoneal (i.p) administration of $75 \mathrm{mg} / \mathrm{kg}$ of ketamine hydrochloride (Hoffman-La Roche, Basel, Switzerland) and $25 \mathrm{mg} / \mathrm{kg}$ of xylazine (Janssen Pharmaceutics, Beerse, Belgium) in $200 \mu \mathrm{l}$ saline. Analgesia was obtained by subcutaneous injection of buprenorphine hydrochloride $0.1 \mathrm{mg} / \mathrm{kg}$ (Schering-Plough Corporation, New Jersey, USA).

Animal model of acute pancreatitis

Anaesthetized mice underwent mildline laparotomy and the second part of duodenum and papilla of vater were identified. Traction sutures (7-0 prolene) were placed one $\mathrm{cm}$ from the papilla. Parallel to the papilla of vater, a small puncture was made through the duodenal wall with a $23 \mathrm{G}$ needle. A non-radiopaque polyethylene catheter (ID $0.28 \mathrm{~mm}$ ) connected to a micro infusion pump (CMA/100, Carnegie Medicin, Stockholm, Sweden) was inserted through the punctured hole in the duodenum and one $\mathrm{mm}$ into the common bile duct. The common hepatic duct was identified at the liver hilum and clamped with a neurobulldog clamp. Infusions of $10 \mu$ of $5 \%$ sodium taurocholate (Sigma, St. Louis, $\mathrm{MO}$, USA) for $10 \mathrm{~min}$ were retrogradly infused into the pancreatic duct. After completion, the catheter was withdrawn and the common hepatic duct clamp was removed. The duodenal puncture was closed with a purse-string suture (7-0 monofilament). The traction sutures were removed and the abdomen was closed in two layers. Animals were allowed to wake up and were given free access to food and water. Radicicol (60 mg/kg, Sigma) or vehicle (phosphate-buffered saline [PBS]) 
was administered i.p. prior to bile duct cannulation. This dose and scheme of administration of the HSP90 inhibitor was based on a previous investigation. ${ }^{2}$ Animals exposed to taurocholate were pretreated with vehicle $(n=8)$ or radicicol $(n=8)$. Control mice undergoing laparotomy and sodium chloride infusion into the pancreatic duct were pretreated with vehicle (Sham $n$ $=8$ ). In separate experiments were mice exposed to taurocholate treated with vehicle $(n=8)$ or radicicol $(n=8) 2 \mathrm{~h}$ after induction of pancreatitis. All animals were killed $24 \mathrm{~h}$ after pancreatitis induction and assessed for all parameters included in this study. Blood was collected from the tail vein for systemic leukocyte differential counts. Blood samples were also collected from the inferior vena cava for determination of serum amylase levels. Pancreatic tissue was removed and kept in two pieces; one piece was snap frozen in liquid nitrogen for biochemical analysis of myeloperoxidase (MPO) and MIP-2 and the other piece was fixed in formalin for later histological analysis. Lung tissue was also harvested for MPO measurements.

Amylase measurements

Amylase was quantified in blood with a commercially available assay (Reflotron $®$, Roche Diagnostics $\mathrm{GmbH}$, Mannheim, Germany).

Systemic Leukocyte counts

Tail vein blood was mixed with Turks solution $(0.2 \mathrm{mg}$ gentian violet in $1 \mathrm{ml}$ glacial acetic acid, $6.25 \% \mathrm{v} / \mathrm{v}$ ) in a 1:20 dilution. Leucocytes were identified as monomorphonuclear and polymorphonuclear cells in a Burker chamber.

MPO activity

All frozen pancreatic and lung tissues were pre-weighed and homogenized in $1 \mathrm{ml}$ mixture (4:1) of PBS and aprotinin

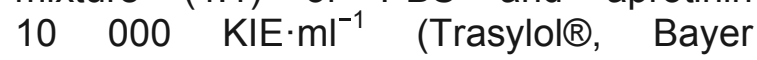
HealthCare AG, Leverkusen, Germany) for $1 \mathrm{~min}$. The homogenate samples were centrifuged $(15339 \times \mathrm{g}, 10 \mathrm{~min})$ and the supernatant was stored at $-20^{\circ} \mathrm{C}$ and the pellet was used for MPO assay as previously described. ${ }^{18}$ All pellets were mixed with $1 \mathrm{ml}$ of $0.5 \%$ hexadecyltrimethylammonium bromide. Next, the sample was frozen for $24 \mathrm{~h}$ and then thawed, sonicated for $90 \mathrm{~s}$, put in a water bath $60^{\circ} \mathrm{C}$ for $2 \mathrm{~h}$, after which the MPO activity of the supernatant was measured. The enzyme activity was determined spectrophotometrically as the MPO-catalysed change in absorbance in the redox reaction of $\mathrm{H}_{2} \mathrm{O}_{2}(450 \mathrm{~nm}$, with a reference filter $\left.540 \mathrm{~nm}, 25^{\circ} \mathrm{C}\right)$. Values are expressed as MPO units.per $\mathrm{g}$ tissue.

Tissue preparation

Pancreas samples were fixed in $4 \%$ formaldehyde phosphate buffer overnight and then dehydrated and paraffin embedded. Six micrometre sections were stained (haematoxylin and eosin) and examined by light microscopy. The severity of pancreatitis was evaluated in a blinded manner by use of a pre-existing scoring system including oedema, acinar cell necrosis, haemorrhage and neutrophil infiltrate on a 0 (absent) to four (extensive) scale as previously described in detail. ${ }^{2}$

MIP-2 levels

MIP-2 levels in serum and pancreas were determined in stored supernatants from homogenized pancreatic tissues. MIP-2 levels were assessed using doubleantibody Quantikine enzyme linked immunosorbent assay kits ( $R$ \& D Systems Europe, Abingdon, UK) using recombinant murine MIP-2 as standard. The minimal detectable protein concentration is less than $0.5 \mathrm{pg} / \mathrm{ml}$.

Flow cytometry assay

Blood was collected $(1: 10$ acid citrate dextrose) from vehicle and pancreatitis mice. To block Fcg III/II receptors and reduce non-specific labeling samples were incubated with an anti-CD16/CD32 for $5 \mathrm{~min}$. Then samples were stained with a PE-conjugated anti-Gr-1 (clone RB68C5, eBioscience, San Diego, CA, USA) antibody. Erythrocytes were lysed and the cells were fixed. Cells were recovered following centrifugation before being analyzed with a FACSCalibur flow 
cytometer (Becton Dickinson, Mountain View, CA, USA). A viable gate was used to exclude dead and fragmented cells. After gating the neutrophil population based on forward and side scatter characteristics, Mac-1 expression was determined on cells positive for $\mathrm{Gr}-1$, which is a neutrophil marker.

Trypsinogen activation in isolated acinar cells

Pancreatic acini cells were prepared by collagenase digestion and gentle shearing as described previously. ${ }^{2}$ Cells were suspended in HEPES-Ringer buffer ( $\mathrm{pH}$ 7.4) saturated with $\mathrm{O}_{2}$ and passed through a $150 \mu \mathrm{m}$ cell strainer (Partec, England). Isolated acinar cells $\left(1 \times 10^{7}\right.$ cells per well) were preincubated with vehicle or radicicol $(200 \mu \mathrm{M}, 30 \mathrm{~min})$ and stimulated with $100 \mathrm{nM}$ cerulein $\left(37^{\circ} \mathrm{C}, 30\right.$ min) in duplicate. The buffer was then discarded and the cells were washed twice with buffer ( $\mathrm{pH}$ 6.5) containing $250 \mathrm{mM}$ sucrose, $5 \mathrm{mM}$ 3-(morpholino) propanesulphonic acid (MOPS) and $1 \mathrm{mM}$ MgSO4. The cells were next homogenized in cold $\left(4^{\circ} \mathrm{C}\right)$, MOPS buffer using a potter Elvejham-type glass homogenizer. The resulting homogenate was centrifuged $(56 \times \mathrm{g}, 5 \mathrm{~min})$, and the supernatant was used for assay. Trypsin activity was measured flourometrically using Boc-GluAla-Arg-MCA as substrate as described previously. ${ }^{21}$ For this purpose, a $200 \mu \mathrm{l}$ aliquot of the acinar cell homogenate was added to a cuvette containing assay buffer (50 mM Tris, $150 \mathrm{mM} \mathrm{NaCl}, 1 \mathrm{mM} \mathrm{CaCl} 2$ and $0.1 \% \mathrm{BSA}, \mathrm{pH} 8.0$ ). The reaction was initiated by the addition of substrate, and the fluorescence emitted at $440 \mathrm{~nm}$ in response to excitation at $380 \mathrm{~nm}$ was monitored. Trypsin levels $(\mathrm{pg} / \mathrm{ml})$ were calculated using a standard curve generated by assaying purified trypsin. Viability of the pancreatic acinar cells was higher than $95 \%$ as determined by trypan blue dye exclusion.

\section{Analysis of data}

Data are presented as mean values \pm SEM. Statistical evaluations were performed by using non-parametrical tests (Mann-Whitney). $P<0.05$ was considered significant and $n$ represents the number of animals.

\section{Results}

Heat shock protein 90 controls tissue damage in pancreatitis

In order to examine the role of heat shock protein 90 in severe $\mathrm{AP}$, serum amylase levels were first examined as an indicator of tissue damage. We observed that retrograde infusion of taurocholate in the pancreatic duct increased serum amylase levels by 5 -fold (Figure 1, $P<0.05$ vs. Sham, $n=8$ ). Treatment with the heat shock protein inhibitor radicicol decreased taurocholate-induced serum amylase levels from $410 \pm 0.61 \mu \mathrm{Kat} / \mathrm{l}$ to $208 \pm$ $0,37 \mu \mathrm{Kat} / \mathrm{l}$, corresponding to a $50.7 \%$ reduction (Figure 1, $P<0.05$ vs. vehicle + taurocholate, $n=8)$.

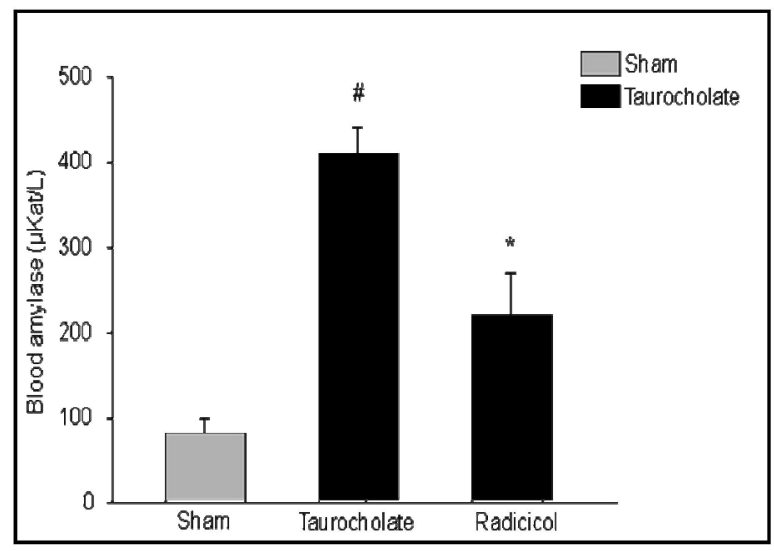

Figure 1: blood amylase $(\mu \mathrm{Kat} / \mathrm{l})$ in sham and taurocholate-exposed mice pretreated with PBS or the HSP 90 inhibitor radicicol $(60 \mathrm{mg} / \mathrm{kg})$. Blood samples were obtained $24 \mathrm{~h}$ after pancreatitis induction. Data represent means \pm SEM and $n=8$. ${ }^{\#} P<0.05$ versus sham and ${ }^{*} P<0.05$ versus PBS + taurocholate. 
Examination of tissue morphology revealed that control mice had a normal pancreatic microarchitecture (Figure 2, $n=8$ ), whereas taurocholate lead to significant destruction of the pancreatic tissue structure characterized by acinar cell necrosis, edema formation and neutrophil accumulation (Figure 2, $n=8$ ). We found that inhibition of heat shock protein 90 protected against taurocholate-provoked tissue destruction (Figure 2, $n=8$ ). For example, it was observed that administration of radicicol reduced taurocholate-evoked acinar cell necrosis by $66.6 \%$ and edema by $52 \%$ in the pancreas (Figure $2 \mathrm{~b}$ and $\mathrm{c}, P<0.05$ vs. vehicle + taurocholate, $n=8$ ). Moreover, radicicol decreased the number of extravascular leukocytes by $57.1 \%$ in pancreatitis mice (Figure 2d, $P<0.05$ vs. vehicle + taurocholate, $n=8)$. Challenge with taurocholate increased the number of circulating MNLs and PMNLs, suggesting on-going systemic activation (Table 1). Inhibition of heat shock protein 90 reversed changes in leukocyte differential counts in the circulation towards the levels in control animals (Table 1).

Table 1: Systemic leukocyte differential counts.

\begin{tabular}{lccc}
\hline & PMNL & MNL & Total \\
\hline Sham & $1.3 \pm 0.3$ & $9.4 \pm 0.3$ & $10.7 \pm 0.6$ \\
PBS + Pancreatitis & $0.9 \pm 0.2^{\#}$ & $6.2 \pm 0.2^{\#}$ & $7.1 \pm 0.4^{\#}$ \\
Radicicol + Pancreatitis & $1.4 \pm 0.1$ & $11.3 \pm 0.3$ & $12.5 \pm 0.4$ \\
\hline
\end{tabular}

blood was collected from sham, saline control, taurocholate-treated animals receiving PBS or the heat shock protein inhibitor radicicol $(60 \mathrm{mg} / \mathrm{kg})$. Cells were identified as monomorphonuclear leukocytes (MNL) and polymorphonuclear leukocytes (PMNL). Data represents mean $\pm S E M$, $10^{6}$ cells/ml and $n=8$. ${ }^{\#} P<0.05$ versus sham and ${ }^{*} P<0.05$ versus $P B S+$ taurocholate.

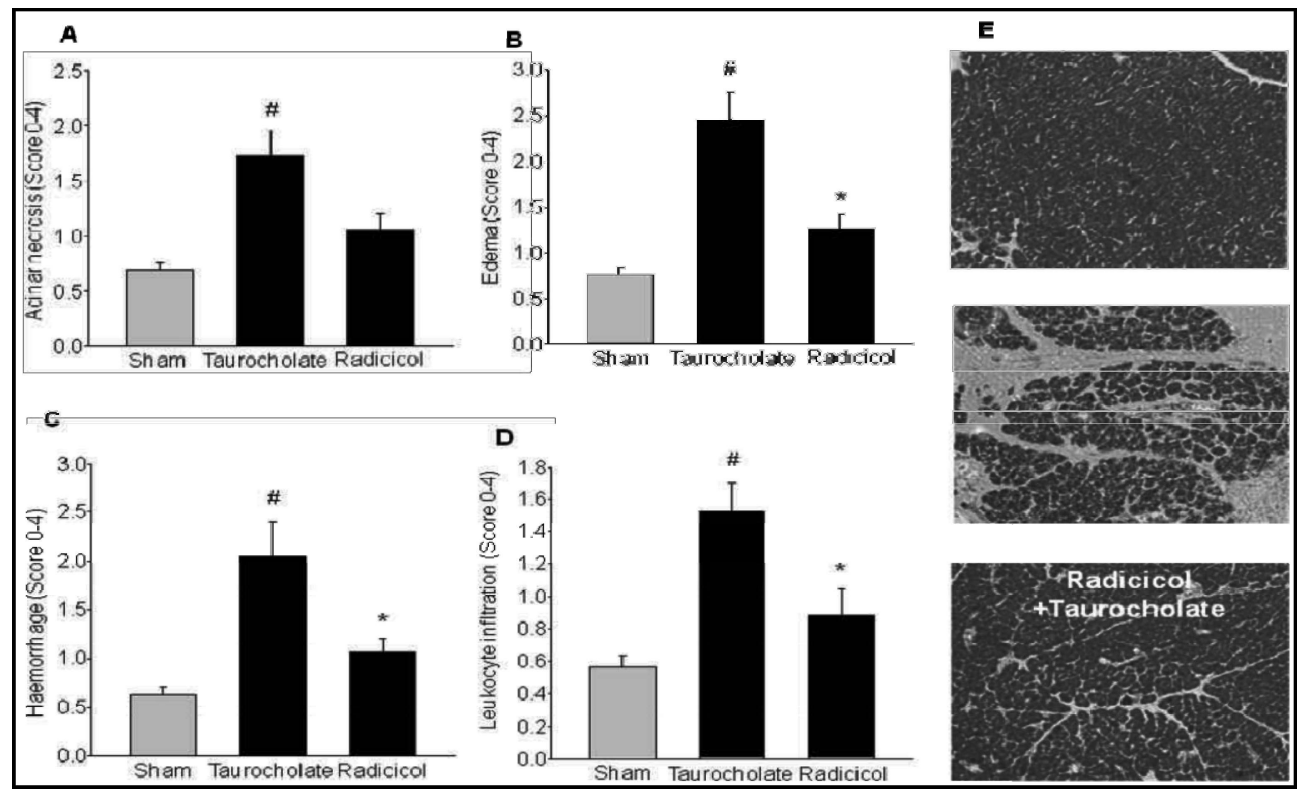

Figure 2: HSP 90 regulates tissue damage in acute pancreatitis. (A) Acinar cell necrosis (B) edema formation (C) hemorrhage and (D) extravascular leukocytes in sham and taurocholate-exposed mice pretreated with PBS or the HSP 90 inhibitor radicicol $(60 \mathrm{mg} /$ $\mathrm{kg}$ ). (E) Representative hematoxylin \& eosin sections of the pancreas. Samples were harvested $24 \mathrm{~h}$ after pancreatitis induction. Bars represent $100 \mu \mathrm{m}$. Data represent means \pm SEM and $n=8$. ${ }^{\#} P<0.05$ versus sham and ${ }^{*} P<0.05$ versus PBS + taurocholate. 

Kurdistan)

Heat shock proteine regulates neutrophil infiltration in pancreatitis

Tissue levels of MPO were used as an indicator of neutrophil infiltration. We found that challenge with taurocholate increased pancreatic MPO activity of by 10 -fold (Figure 3a, $P<0.05$ vs. Sham, $n=8$ ). Inhibition of heat shock protein 90 inhibition reduced taurocholate-provoked pancreatic levels of MPO by $27 \%$ (Figure 3a, $P<0.05$ vs. vehicle + taurocholate, $n=8$ ).
As part of a systemic inflammatory response in severe AP, activated neutrophils accumulate in the pulmonary microvasculature. Indeed, it was observed that challenge with taurocholate markedly enhanced the MPO activity in the lung. Heat shock protein 90 inhibition reduced MPO levels in the lung by more than $30.7 \%$ in mice challenged with taurocholate (Figure $3 \mathrm{~b}, P<0.05$ vs. vehicle + taurocholate, $n=8)$.

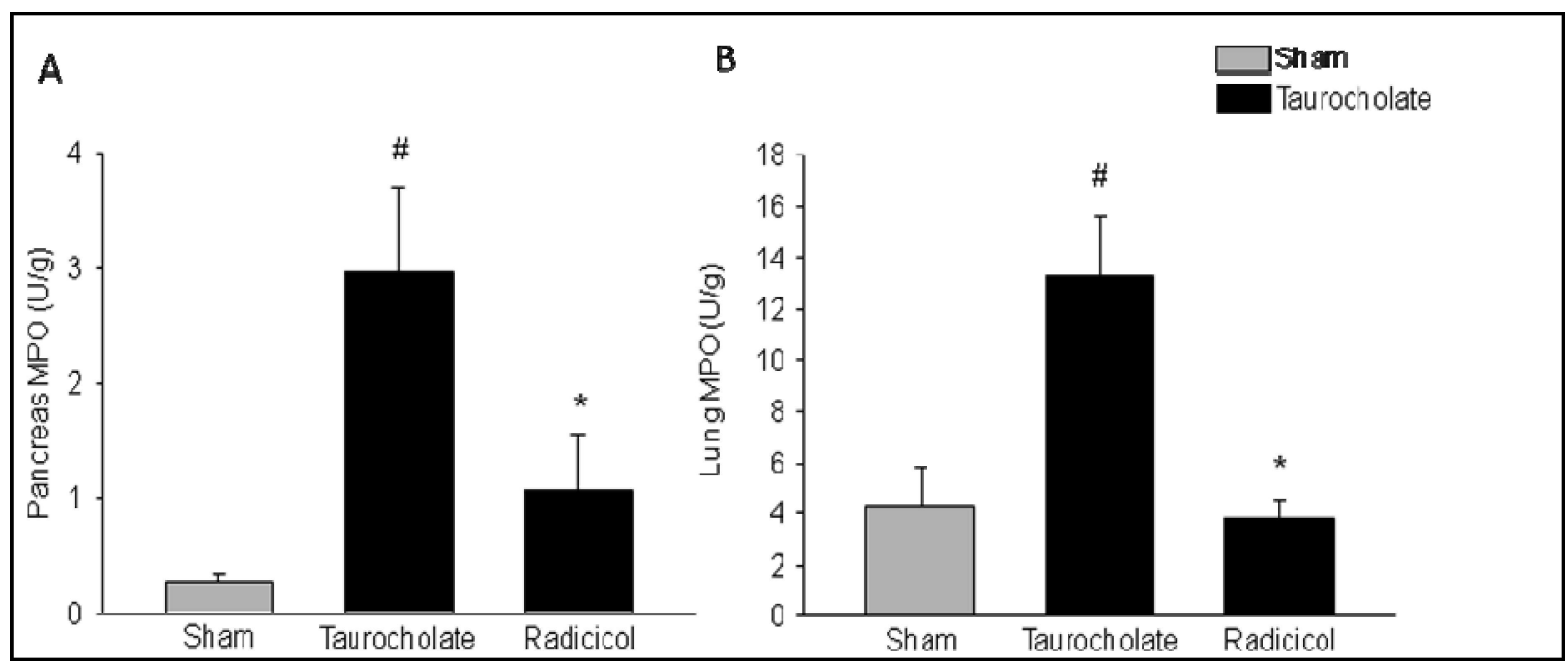

Figure 3: HSP 90 controls taurocholate-induced neutrophil accumulation. MPO levels in the (A) pancreas and (B) lung in sham and taurocholate-exposed mice pretreated with PBS or the HSP 90 inhibitor $(60 \mathrm{mg} / \mathrm{kg})$. Samples were harvested $24 \mathrm{~h}$ after pancreatitis induction. Data represent means \pm SEM and $n=8 .{ }^{\#} P<0.05$ versus sham and ${ }^{*} P<0.05$ versus PBS + taurocholate. 
In addition, we observed that challenge with taurocholate markedly increased MIP-2 levels in the pancreas from $0.08 \pm 0.04$ to $8.8 \pm 0.05 \mathrm{pg} / \mathrm{mg}$ (Figure 4, $P<0.05$ vs. Sham, $n=8$ ). Administration of radicicol had no significant effect on MIP -2 levels in the inflamed pancreas (Figure 4 $P>0.05$ vs. vehicle + taurocholate, $n=8$ ). Moreover, we noted that Mac-1 expression was increased on the surface of neutrophils in mice with pancreatitis (Figure 5a, $P<0.05$ vs. Sham, $n=8$ ), indicating systemic activation in this experimental model. Inhibition of heat shock protein 90 signaling markedly reduced neutrophil expression of Mac-1 in pancreatitis (Figure 5a, $P<0.05$ vs. vehicle + taurocholate, $n=8$ ). In order to test whether this inhibitory effect of radicicol on neutrophil expression of Mac-1 was indirect or direct, we stimulated isolated neutrophil with MIP-2 in vitro and found that radicicol markedly decreased MIP-2-induced upregulation of Mac-1 on isolated neutrophils (Figure $5 \mathrm{~b}, P<0.05$

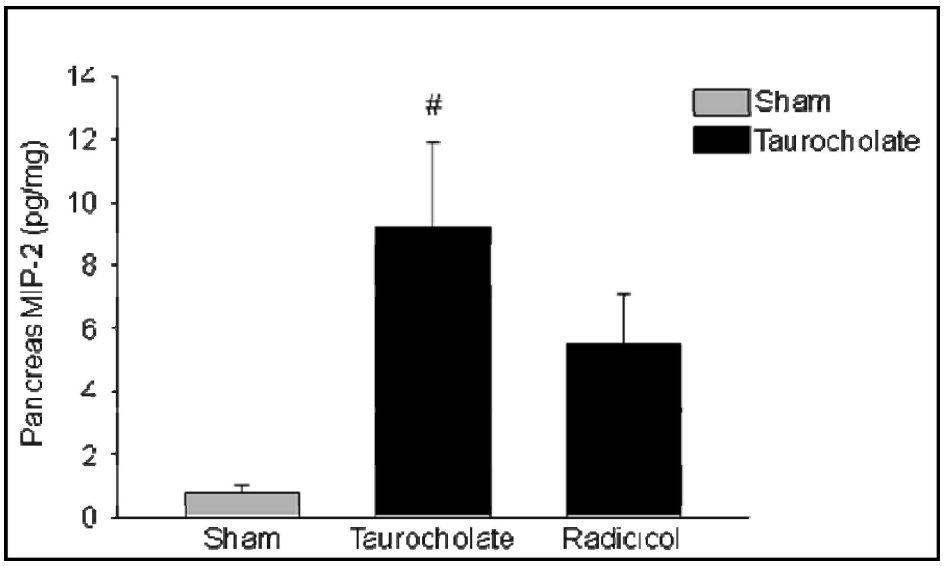

Figure 4: Chemokine formation in the pancreas. Pancreatic levels of MIP-2 were determined in sham and taurocholate-exposed mice pretreated with PBS or the HSP 90 inhibitor $(60 \mathrm{mg} / \mathrm{kg})$. Samples were harvested $24 \mathrm{~h}$ after pancreatitis induction. Data represent means \pm SEM and $n=8$. ${ }^{\#} P<0.05$ versus sham and ${ }^{*} P<0.05$ versus PBS + taurocholate.

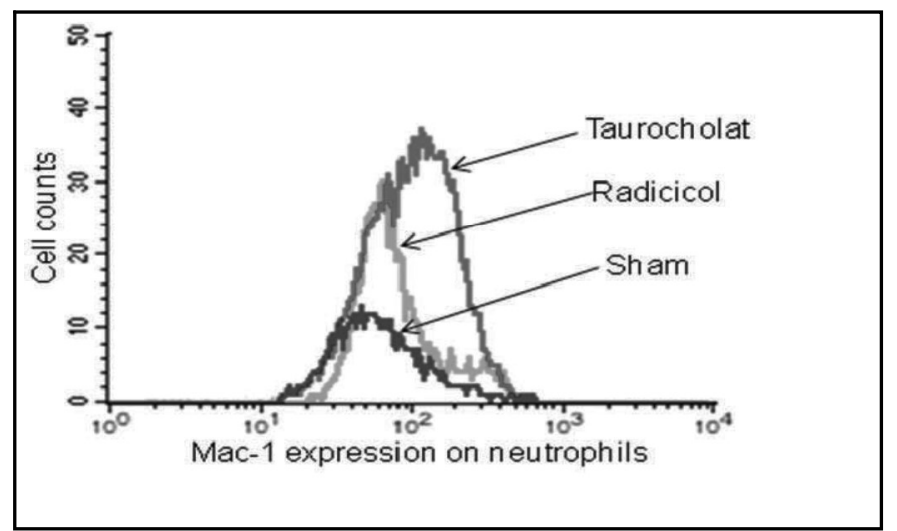

Figure 5: HSP 90 regulates Mac-1 expression on neutrophils. Mac-1 expression $(A+B)$ on neutrophils in PBS and radicicol $(60 \mathrm{mg} / \mathrm{kg})$ treated animals $24 \mathrm{~h}$ after induction of pancreatitis and $(C+D)$ on isolated neutrophils incubated with MIP-2 $(5 \mu \mathrm{g} / \mathrm{ml})$ and PBS or radicicol $(100 \mu \mathrm{M})$. Fluorescence intensity is shown on the $\mathrm{x}$-axis and cell counts on the $y$-axis. Histograms are representative of 8 samples. Data represent means \pm SEM and $n=8$. ${ }^{\#} P<0.05$ versus sham and ${ }^{*} P<0.05$ versus $P B S+$ taurocholate. 
Trypsinogen activation in acinar cells in vitro

We nest asked whether heat shock protein 90 may regulate trypsinogen activation in pancreatic acinar cells in vitro. For this purpose, we isolated acinar cells from the pancreas of mice and incubated the cells with cerulein. It was found that cerulein stimulation increased trypsinogen activation by more than 30 -fold compared to unstimulated cells (Figure $7, P<0.05$ vs. control, $n=5$ ). However, preincubation of the acinar cells with radicicol had no effect on secretagogue-induced activation of trypsinogen (Figure 6, $P<0.05$ vs. vehicle + cerulein, $n=5$ ).

\section{Discussion}

Signaling cascades controlling proinflammatory pathways in pancreatitis are incompletely understood. Our present study demonstrates for the first time that heat shock protein 90 is an important regulator of the pathophysiology in severe AP. These results demonstrate that heat shock protein 90 is involved in the surface upregulation of Mac-1 on neutrophils. Inhibition of heat shock protein 90 activity not only decreases neutrophil infiltration in the pancreas but also attenuates acinar cell necrosis and serum amylase levels in AP. In addition, we found that heat shock protein 90 inhibition abolished accumulation of neutrophils in the lung, suggesting that heat shock protein 90 controls both local and systemic inflammation in severe AP. Heat shock protein 90 activity has been reported to control pro-inflammatory actions in experimental models of multiple sclerosis and sepsis. ${ }^{7}$ Herein, we found that heat shock protein 90 inhibition with a specific heat shock protein 90 inhibitor (Radicicol) significantly reduced tissue injury in severe AP. For example, administration of radicicol decreased taurocholate-provoked increase in blood amylase and acinar cell necrosis suggesting that heat shock protein 90 activity regulates a significant part of the tissue damage in severe AP.

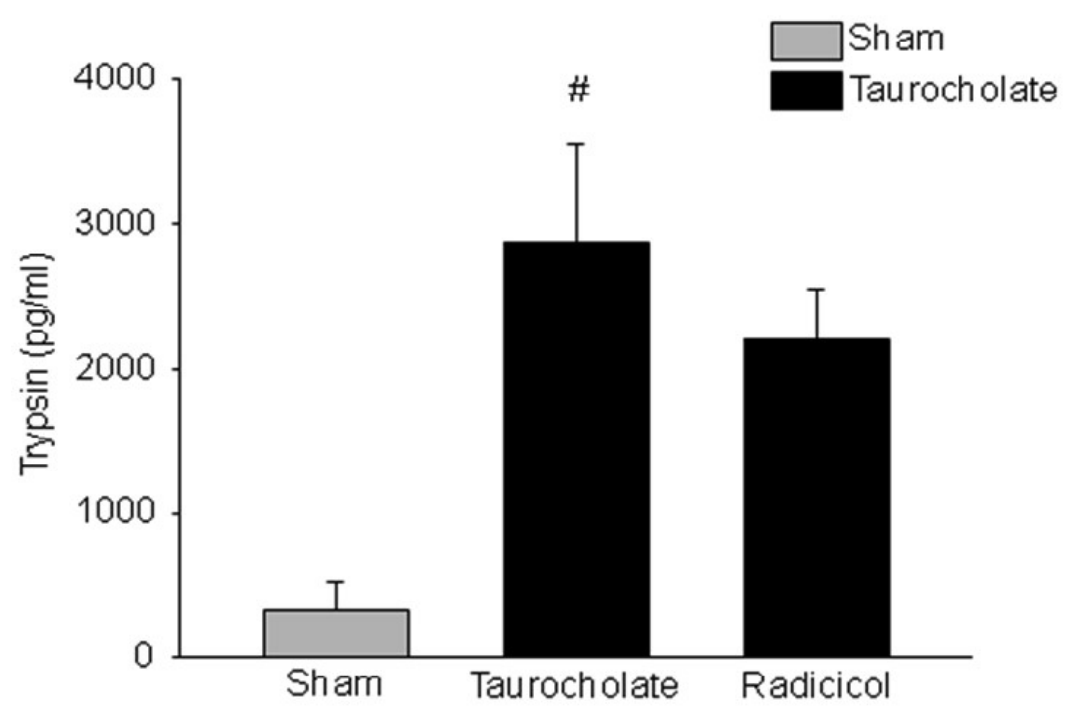

Figure 6. Acinar cell activation of trypsinogen was measured in negative control cells and cerulein-exposed acinar cell homogenate pretreated with PBS or radicicol $(200 \mathrm{mM})$. Activation of trypsinogen activation was quantified by measuring enzymatic activity of trypsin fluorometrically by using Boc-GIn-Ala-Arg-MCA as the substrate as described in detail in Materials and Methods. Trypsin levels $(\mathrm{pg} / \mathrm{ml})$ were calculated using a standard curve generated by assaying purified trypsin. Data represent means $\pm S E M$ and $n=8$. ${ }^{\#} \mathrm{P}<0.05$ versus PBS control. 
These data constitutes the first evidence in the literature that inhibition of the heat shock protein 90 signaling pathway protects against severe AP. In this context, it is interesting to note that HSP, which are mainly used to regulate severe levels in patients with cardiovascular diseases, have been reported to attenuate experimental pancreatitis. $^{8} \quad$ It is widely held that neutrophil infiltration is a prominent feature in pancreatitis. ${ }^{9}$ For example, neutrophil depletion has repeatable been reported to ameliorate tissue damage in $\mathrm{AP}^{9}{ }^{9}$ In the present study, we found that taurocholate challenge markedly enhanced MPO activity and the number of extravascular neutrophils in the pancreas. Administration of radicicol significantly reduced MPO levels and the number of extravascular neutrophils in the pancreas, indicating that heat shock protein 90 activity is an important regulator of neutrophil recruitment in the inflamed pancreas. Considering the critical role of neutrophils in the pathophysiology of pancreatitis. ${ }^{2}$ it might be forwarded that the inhibitory effect of radicicol on neutrophil responses might explain the protective effect of radicicol in AP. In addition, systemic complications of severe AP include pulmonary accumulation of neutrophils. ${ }^{10}$ We found that lung levels of MPO clearly increased in response to taurocholate challenge. Interestingly, radicicol decreased pulmonary MPO activity, suggesting that heat shock protein 90 also regulates systemic activation and infiltration of neutrophils in the lung in severe AP. Numerous studies have shown that specific adhesion molecules control the extravasation process of leukocytes. ${ }^{2}$ Although the detailed role of certain adhesion molecules in facilitating leukocyte accumulation in the pancreas is relatively unclear, several reports have documented that shown that Mac-1 is a dominating molecule in mediating tissue infiltration of neutrophils. ${ }^{5}$ Herein, it was observed that Mac-1 expression on neutrophils increased in response to taurocholate administration.
Notably, treatment with radicicol greatly decreased neutrophil expression of Mac-1, suggesting that heat shock protein regulates Mac-1 expression on neutrophils in AP. We next asked whether this inhibitory effect of radicicol might be indirect and related to formation of CXC chemokines, such as MIP-2, which is a particularly potent activator of neutrophils. ${ }^{9}$ It was therefore of great interest to examine formation of MIP-2 in the pancreas in this study. It was found that taurocholate provoked a significant increase in MIP-2 levels in the pancreas. However, inhibition of heat shock protein 90 had no effect on taurocholate-induced production of MIP-2 in the pancreas. We next asked whether radicicol might directly inhibit Mac-1 upregulation on activated neutrophils. Indeed, we found that radicicol abolished MIP-2-induced upregulation of Mac-1 on the surface of isolated neutrophils, suggesting that heat shock protein 90 directly regulate Mac-1 expression in neutrophils. Trypsinogen activation is generally considered as a central feature in the pathophysiology of AP. ${ }^{1}$ A recent study showed that GTpase signaling regulates trypsinogen activation in acinar cells. ${ }^{5}$ However, we observed that Inhibition of heat shock protein 90 activity had no effect on secretagogoue-induced activation of trypsin in isolated acinar cells in vitro. The precise role of HSP in AP needs to be addressed in future studies. Nonetheless, these findings suggest that the protective effects of HSP90 are downstream of trypsin activation in pancreatitis. This notion is in line with the concept in the present study suggesting that a dominant role of $\mathrm{fHSP}$ in AP is related to inhibition of Mac-1 expression and neutrophil accumulation in the pancreas and lung. In conclusion, these findings demonstrate that HSP 90 signaling regulates tissue damage in severe AP. Our results show that inhibition of HSP 90 attenuates neutrophil expression of Mac-1 and infiltration in the pancreas and the lung, suggesting that HSP 90 controls both 
local and systemic inflammation in pancreatitis. Thus, these data not only delineate a key signaling mechanism in AP but also indicate that targeting HSP might be an effective way to ameliorate to pathological inflammation in severe AP.

\section{Conclusion}

These results demonstrate that heat shock protein 90 signaling plays a significant role in acute pancreatitis by regulating neutrophil infiltration and tissue injury via expression of Mac-1 on neutrophils. Thus, our findings not only elucidate signaling mechanisms in pancreatitis or lung injury but also suggest that heat shock protein might constitute a novel target in the management of severe AP related with lung injury.

\section{Conflict of interest \\ The author declares no competing interests.}

\section{References}

1. Merza M, Awla D, Hwaiz R, Rahman M, Appelros $S$, Abdulla A, et al. Farnesyltransferase regulates neutrophil recruitment and tissue damage in acute pancreatitis Pancreas 2014; 43(3):427-35.

2. Merza M. Isoprenylation and NET formation in acute pancreatitis. PhD. Thesis book, Lund University 2015.

3. Merza M, Wetterholm E, Zhang S, Regner S, Thorlacius $H$. Inhibition of geranylgeranyltransferase attenuates neutrophil accumulation and tissue injury in severe acute pancreatitis. JLB 2013; 94(3):493-502.

4. Madhi R, Rahman M, Taha D, Linders J, Merza M, Wang $Y$, et al. Platelet IP6K1 regulates neutrophil extracellular trap-microparticle complex formation in acute pancreatitis. JCl insight 2019.

5. Wetterholm E, Linders J, Merza M, Regner S, Thorlacius $\mathrm{H}$. Platelet-derived CXCL4 regulates neutrophil infiltration and tissue damage in severe acute pancreatitis. Translational Research 2015; 176:105-18.

6. Merza M, Rahman M, Zhang S, Hwaiz R, Regner S, Schmidtchen A, et al. Human thrombin -derived host defense peptides inhibit neutrophil recruitment and tissue injury in severe acute pancreatitis AJPGLP 307(9):914-21.

7. Zhao Y, Huang ZJ, Rahman M, Luo Q, Thorlacius $\mathrm{H}$. Radicicol, an Hsp90 inhibitor, inhibits intestinal inflammation and leakage in abdominal sepsis. J Surg Res 2013; 182(2):312-8.
8. Boudesco C, Cause S, Jego G, Garrido C. Hsp70: A Cancer Target Inside and Outside the Cell. Methods Mol Biol 2018;1709:371-96.

9. Merza M, Hartman $H$, Rahman M, Hwaiz R, Zhang E, Renström E, et al. Neutrophil extracellular traps induce trypsin activation, inflammation, and tissue damage in mice with severe acute pancreatitis 2015 Gastroenterology; 149(7):1920-31.

10. Abdulla A, Awla D, Thorlacius H, Regnér S. Role of neutrophils in the activation of trypsinogen in severe acute pancreatitis 2012 JLB; 90(5):97582. 